\title{
Sensory Transduction in Peripheral Nerve Axons Elicits Ectopic Action Potentials
}

\author{
Tal Hoffmann, ${ }^{1}$ Susanne K. Sauer, ${ }^{1}$ Raymund E. Horch, ${ }^{2}$ and Peter W. Reeh ${ }^{1}$ \\ ${ }^{1}$ Institute for Physiology and Pathophysiology, and ${ }^{2}$ Department of Plastic and Hand Surgery, University Medical Center, University of Erlangen- \\ Nuremberg, D-91054 Erlangen, Germany
}

\begin{abstract}
Sensory properties of unmyelinated axons in the isolated rat sciatic nerve have been revealed previously by measuring stimulated neuropeptide release in response to noxious stimuli. In addition, axonal sensitization by inflammatory mediators has been demonstrated and shown to depend on the heat- and proton-activated ion channel transient receptor potential vanilloid receptor-1. It was unclear whether this responsiveness is accompanied by ectopic generation of action potentials, which may play a crucial role in painful neuropathies. We explored this hypothesis using the isolated mouse skin-nerve preparation. This method enabled us to directly compare the sensory properties of axons in the peripheral nerve with their characterized cutaneous terminals in the receptive field using propagated action potentials as an index of axonal activation. Single-fiber recordings from 51 mechanosensitive mouse C-fibers revealed that a majority of the polymodal nociceptors responded with an encoding discharge rate to graded heating of the cutaneous receptive field $(n=$ 38 ) as well as of the saphenous nerve carrying the fiber under investigation $(n=25 ; 66 \%)$. Axonal heat responses paralleled those of the receptive fields with regard to thresholds and discharge rates $\left(41.5 \pm 4.3^{\circ} \mathrm{C} ; 7.7 \pm 9.6\right.$ spikes in a $20 \mathrm{~s} 32-48^{\circ} \mathrm{C}$ ranged stimulation). In contrast, axonal mechanosensitivity was poor and noxious cold sensitivity more rarely encountered. In conclusion, peripheral nerve axons exhibit sensory transduction capacities similar to their nociceptive terminals in the skin with respect to noxious heat, although not to mechanical and cold sensitivity. This may become a source of ectopic discharge and pain if axonal heat threshold drops to body temperature, as may be the case during inflammation-like processes in peripheral nerves.
\end{abstract}

Key words: heat sensitivity; unmyelinated fibers; nociception; sensory neurons; neuropathy; bradykinin

\section{Introduction}

Unmyelinated axons of peripheral nerves have been shown previously to develop various nociceptive properties at the proximal stump after transection (axotomy). These sensory properties included the development of spontaneous ectopic discharge (Michaelis et al., 1995), axonal sensitivity to a variety of algesic agents such as bradykinin (BK), prostaglandin $\mathrm{E} 2$, acidic $\mathrm{pH}$, and capsaicin (Michaelis et al., 1997; Moalem et al., 2005), and the appearance of mechanosensitivity and thermosensitivity along the axon itself at the developing neuroma site (Koschorke et al., 1991; Michaelis et al., 1995, 1997; Blenk et al., 1996). The common hypothesis explaining these observations is that anterograde axonal transport of sensory transducer molecules such as heatactivated channels or of voltage-gated ion channels causes an accumulation of these membrane proteins at the lesioned site. Translocation of the receptors and channels to the axonal membrane could provide the regenerating axonal sprouts with the same properties their sensory nerve endings possessed before axotomy (Devor and Govrin-Lippmann, 1983; Guharay and Sa-

Received Dec. 13, 2007; revised May 7, 2008; accepted May 13, 2008.

This work was supported by German-Israeli Foundation Grant GIF 1-772-252.1/2002 (P.W.R.)

Correspondence should be addressed to Tal Hoffmann, Institute for Physiology and Pathophysiology, University

of Erlangen-Nuremberg, Universitaetsstrasse 17, D-91054 Erlangen, Germany. E-mail: diskin@ physiologie1.uni-erlangen.de.

DOI:10.1523/JNEUROSCI.1627-08.2008

Copyright $\odot 2008$ Society for Neuroscience $\quad$ 0270-6474/08/286281-04\$15.00/0 chs, 1984; Koschorke et al., 1991, 1994; Blenk et al., 1996; Michaelis et al., 1999).

The question arose whether the sensory properties observed in regenerating axons truly rely on de novo-born mechanisms or represent sensory properties present in the axonal membrane at all times. Indirect evidence obtained from second-order neurons in the cat as well as in vivo single-fiber recordings in rats suggest that there may be a certain heat sensitivity in normal C-axons of peripheral nerves (Zimmermann and Sanders, 1982; Blenk et al., 1996). In addition, the isolated desheathed sciatic nerve of the rat responds to noxious heat with a well graded and calciumdependent release of the neuropeptide calcitonin gene-related peptide (CGRP) expressed in C- and A $\delta$-fibers (Sauer et al., 2001). The stimulus-response relationship in this preparation shows a high thermal coefficient, $Q_{10}$ of 15 , which, together with the above electrophysiology, is highly suggestive of underlying nociceptive transduction mechanisms.

We have scrutinized the question of axonal sensitivity using an isolated mouse skin-nerve preparation. The single-fiber recordings enabled us to quantify and directly compare, for the first time, the sensory properties of individual axons in the peripheral nerve stem with their characterized cutaneous terminals in the receptive field $(\mathrm{RF})$.

\section{Materials and Methods}

Animals. The study was performed on male and female inbred C57BL/6 mice weighing 20-25 g. Animals were killed in a pure $\mathrm{CO}_{2}$ atmosphere. 
Table 1. Summary of the single-fiber recordings

\begin{tabular}{lllll}
\hline Stimulation & Mechanical (von Frey) & Heat $\left(48^{\circ} \mathrm{C}\right)$ & Cold $\left(4^{\circ} \mathrm{C}\right)$ & BK $(10 \mu \mathrm{M})$ \\
\hline Fibers responsive in the receptive field & 51 & 38 & 8 & $85 \%^{a}$ \\
Fibers responsive also in the axon & 7 & 25 & 2 & 2 of 13 \\
Proportion of fibers sensitive in both RF and axon & $11 \%$ & $65.7 \%$ & $25 \%$ & $15 \%$ \\
\hline
\end{tabular}

Fibers responsive in their endings and axons to mechanical and thermal stimuli are shown. The numbers listed in the BK column represent the share of BK-sensitized axons out of 13 tested fibers.

${ }^{a}$ Although only the axons were examined in the present study, $85 \%$ of the nerve endings are principally known to express $B_{2}$ receptors and respond to BK with discharge activity and a huge prevalence of heat sensitization (Liang et al., 2001 ).

Receptive field stimulation. Single-fiber recordings from mechanoreceptive C-fibers of the saphenous nerve were obtained using the isolated skin-nerve preparation as described previously (Reeh, 1986). The mechanical threshold of the fibers was characterized using a custom-made set of novel von Frey probes. Each probe consisted of a hand-held aluminum pipe closed at its lower end by a plug of polyoxymethylene (Delrin) with a center bore hole slightly wider than a 27 -gauge cannula that could slide inside with low friction. The upper end of the cannula bore different weights creating weight forces of $1-128 \mathrm{mN}$ calibrated according to a geometric scale $(n=15)$. The lower blunted end had a rounded tip of nail varnish $(0.6 \mathrm{~mm}$ diameter $)$. When perpendicularly applied, these probes allowed for reproducible instant forces, much like the traditional von Frey bristles.

For thermal stimulation, an oval isolating metal ring was placed on the $\mathrm{RF}$, evacuated from fluids, and feedback-controlled radiant heat $(20 \mathrm{~s}$ ramp of $32-48^{\circ} \mathrm{C}$ ) as well as cold (topical administration of ice-cold buffer) stimulations were applied to the epidermal and corium sides of the skin, respectively. The thermal threshold for each fiber was defined as the temperature at the second spike discharge.

Axonal stimulation. The axon from an identified RF was electrically stimulated in the saphenous nerve stem using a metal microelectrode. The electrostimulation was then combined with mechanical stimulation to the RF to assure the identical origin of the electrically and mechanically evoked action potentials using the marking technique (Schmelz et al., 1995). Axonal mechanosensitivity was examined using a special von Frey probe set. These probes were identical to the probes used for the evaluation of mechanosensitivity in the nerve endings, except instead of rounded tips, they were equipped with 0.5 - $\mathrm{mm}$-diameter cylindrical cross bars. The bars were pushed against the convex nerve stem orthogonally to the nerve axis at various points along its course forming punctate contact and, thus, passing the calibrated weight force onto the nerve while avoiding sideward slipping of the probe. The cross bars also ensured conductance of the calibrated weight force to all of the fibers inside the tested nerve stem, regardless of their relative location. The metal ring was then placed over the nerve, making sure it kept a clear distance to the border of the RF (on average, $7 \pm 2.9 \mathrm{~mm}$; range, $4-18 \mathrm{~mm}$ ), a miniature thermocouple was placed on top of the nerve, and thermal sensitivity was tested as in the RF. In control experiments $(n=5)$ using two thermocouples, a peak temperature of $46^{\circ} \mathrm{C}$ was measured on the nerve, whereas just outside of the metal ring the intracutaneous temperature peaked at $37.2 \pm 0.5^{\circ} \mathrm{C}$. At a $4 \mathrm{~mm}$ distance to the wall of the ring, a minimal temperature change of $3^{\circ} \mathrm{C}\left(32\right.$ to $\left.35^{\circ} \mathrm{C}\right)$ was to be measured because of the sharp-edged focus of the parabolic projector lamp used for radiant heat stimulation.

Bradykinin (Sigma) at a concentration of 10 or $100 \mu \mathrm{M}$ was applied to some of the heat-responsive nerve branches between two consecutive heat stimuli (5 min interval).

\section{Results}

A total of 51 mechanosensitive primary afferent C-fibers with RFs in the isolated mouse skin were studied. The mean conduction velocity of the fibers was $0.46 \pm 0.1 \mathrm{~m} / \mathrm{s}( \pm \mathrm{SD})$. Of these, $33 \mathrm{RFs}$ were also heat sensitive [ $\mathrm{C}$ mechano-heat-sensitive $(\mathrm{CMH})$ fiber], three were also cold sensitive [ $\mathrm{C}$ mechano-cold-sensitive (CMC) fiber], five units also responded to both heat and cold stimulation [C mechano-heat-cold-sensitive (CMHC) fiber], and 10 showed only mechanical responsiveness (Table 1).

\section{Axonal mechanosensitivity}

A total of seven fibers of the 51 tested exhibited certain mechanical responsiveness to nerve-stem stimulation. This proportion is comparable with the number of mechanically sensitive C-fiber axons found by Dilley and Bove (2008) (3 of 43), who demonstrated that blocking axoplasmic transport increases the prevalence of axonal mechanosensitivity proximal of the block (to 11 of 36). The von Frey threshold of the individual axon was always much higher than that of the RF. The median von Frey threshold measured in the RFs of the seven units was $5.7 \mathrm{mN}$ (range, 4-45.3 $\mathrm{mN}$ ) and a median threshold of $45.3 \mathrm{mN}$ (ranging from 22.6 to $90.5 \mathrm{mN}$ ) was tentatively determined for the mechanosensitive axons. The threshold responses of the axons were hardly reproducible and stronger stimuli (two to three times the von Frey threshold) did not reliably evoke a higher-frequency discharge which, if present, poorly corresponded to onset and offset of the stimulation. These properties were in major contrast to those recognized in the RFs of the same fibers which, in principle, are capable of truly encoding noxious mechanical stimuli with respect to timing and intensity (Mogil et al., 2005).

No afterdischarge was observed in any of the above recordings. The scarcity and irregularity of the axonal mechanosensitivity precluded further detailed analysis. Notably, even after applying strong glass rod pressure to the nerve stem, the mechanosensitivity of the RF was unchanged.

\section{Axonal thermal sensitivity and sensitization}

Two of the three CMC units responded to axonal cold stimulation with a prompt and adapting burst of spikes. These responses appeared to be similar to the discharge evoked in the RF, however, the small number of cold-sensitive fibers encountered as well as the small magnitude of the cold responses prevented additional analysis.

In contrast, two thirds of the $\mathrm{CMH}(\mathrm{C})$ fibers tested (25 of 38) exhibited a clear and reproducible heat sensitivity in both RF and axon (Fig. 1). The average first heat threshold was $42.5 \pm 3.6^{\circ} \mathrm{C}$ for the RFs and $41.5 \pm 4.3^{\circ} \mathrm{C}$ for the axons. The mean heatinduced discharge from the RFs of these $25 \mathrm{CMH}$ fibers rose with temperature in an approximately log-linear way, as published previously (Zimmermann et al., 2005). The first axonal heat stimulation led to a less consistent increase in firing rate; the discharge pattern was reminiscent of a greater tendency toward adaptation in the nerve than in the RFs. The second axonal heat response paralleled the RF response with the only difference being a smaller mean number of spikes (axon, 5.5 spikes/20 s; RF, 11.5 spikes/20 s; $p<0.01$, Wilcoxon matched pairs test). We entered the heat thresholds and response magnitudes of both RFs and nerves into a correlation matrix and found no correlation in heat responsiveness between both sites after the first and second heat stimulation ( $r=-0.074$ and $r=-0.435$, respectively). This supports the notion that the RF and nerve stem responses originated from two distinct and independently stimulated sites.

Heat-induced axonal CGRP release from the desheathed pe- 

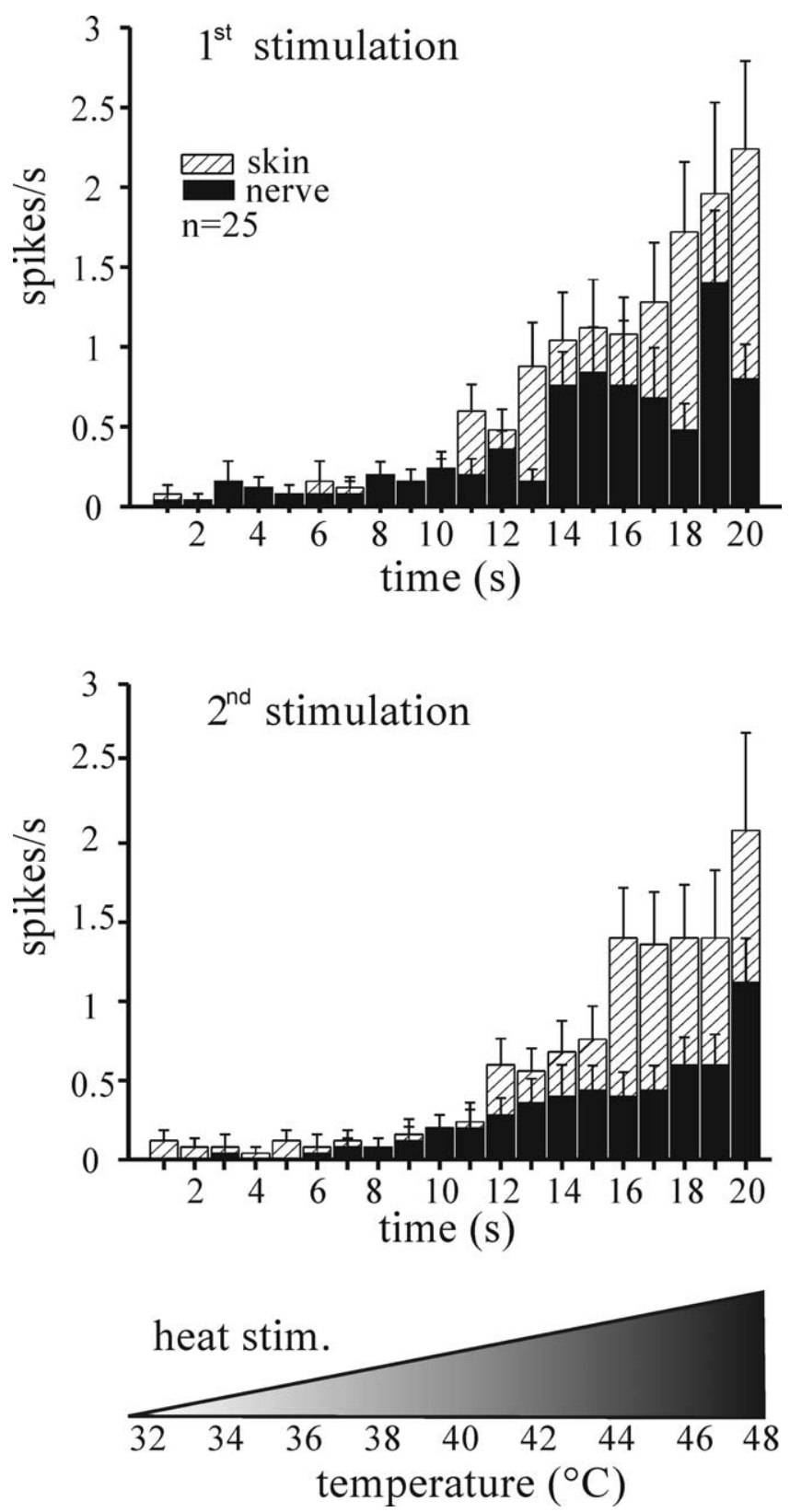

Figure 1. Cutaneous versus axonal heat response in isolated mouse skin. The histograms show averaged heat-induced spike discharge recorded from 25 (of 38) CMH fibers exhibiting axonal heat sensitivity. Two consecutive heat stimuli were applied with a 5 min interval. Error bars indicate SEM.

ripheral nerve has recently been shown to be sensitized by BK, although BK itself did not evoke any CGRP release (Fischer and Reeh, 2007). We therefore tested for axonal heat sensitization in response to BK superfusion ( $10 \mu \mathrm{M}$ for $5 \mathrm{~min}$ ), although the nerve could not be desheathed to improve diffusion. Only 2 of 13 fibers tested showed a, however marked, change in heat-induced axonal discharge, increasing the total number of spikes in $20 \mathrm{~s}$ by more than twofold (data not shown). The heat threshold remained unchanged. BK alone had no excitatory effect when applied to the intact nerve stem. The mere repetition of the moderate heat stimuli did not cause a major sensitization of the averaged fiber population (Fig. 1).

In summary, although the scarcity of axonal cold responses excluded any further evaluation, axonal heat sensitivity appeared well developed and capable of encoding noxious temperatures although with lower discharge rates than after RF stimulation. It is noteworthy that although all mechanosensitive fibers were subjected to axonal heat and cold stimulation, only the ones thermally responsive in their RFs exhibited a corresponding thermal responsiveness also in their axons (Table 1).

\section{Discussion}

Our results indicate that intact unmyelinated peripheral nerve axons possess specific thermosensory properties that resemble those of their individual cutaneous nociceptive terminals, at least with respect to noxious heat sensitivity in the mouse. The fact that mechanical stimulation of the nerve evoked only a poor and erratic response could be interpreted as a lack of specific mechanosensory transduction in these axons. Strong deformation of axons may still depolarize their membrane and result in sporadic action potentials. In contrast, specific axonal cold transduction may well exist, although our few results were suggestive rather than conclusive.

Apart from the evidence for heat-induced axonal CGRP release (see Introduction), axonal heat sensitivity has previously been suggested from electrophysiological experiments. Recordings obtained from cat dorsal horn neurons showed an increase in ongoing discharge rate when noxious heat was applied to the peripheral nerve of the segmentally corresponding dermatome (Zimmermann and Sanders, 1982). More directly, in vivo singlefiber recordings from the rat sural nerve revealed five (of 172, $\sim 3 \%$ ) not further characterized C-fibers that "could be activated by warm stimuli" (interface temperature median, $47^{\circ} \mathrm{C}$ for $30 \mathrm{~s}$ ) applied to the nerve stem in situ through a contact thermode (Blenk et al., 1996). In our study, radiant heat was passed through the nerve stem to reach $48^{\circ} \mathrm{C}$ on the opposite side where the thermocouple was placed for feedback. The previous underestimation of axonal heat sensitivity may still have other than methodological reasons, such as the relatively high numbers of sympathetic efferents $(\sim 24 \%)$ and heat-insensitive C-fibers in the sural nerve that is a mixed sensory and motor nerve (Baron et al., 1988). In contrast, our experiments included only identified afferent fibers terminating in a mechano-heat-sensitive cutaneous $\mathrm{RF}$, which most likely increased our probability of encountering heat-sensitive axons.

Although our findings strongly argue for the expression of heat-transducing proteins along the axonal membrane, the heatactivated ion channel transient receptor potential vanilloid receptor-1 (TRPV1) alone is most likely not accountable for the axonal heat sensitivity we observed. Despite the fact that TRPV1 receptors are expressed in the axonal membrane (Bernardini et al., 2004) and that TRPV1 is sensitized in both axons and nerve endings when an agonist or inflammatory mediators are applied, it is neither responsible for the basal heat-induced discharge of cutaneous nerve endings nor for the basal heat-induced axonal CGRP release (Zimmermann et al., 2005; Fischer and Reeh, 2007). Thus, other heat-transducing channels are most likely involved in axonal heat sensitivity. One possible candidate might be the heat-inactivated two-pore domain inwardly rectifying (TWIK)-related $\mathrm{K}^{+}$channel (TREK-1), which has been proposed recently to be involved in the heat sensitivity of cutaneous nerve endings in the mouse (Alloui et al., 2006).

Heat and cold responsiveness require both sensory transduction and action potential generation, the latter enabling singlefiber recording. In this respect, axons and nerve endings differ essentially in that C-fibers, in contrast to terminals, do not express the slow inactivating and rapidly repriming TTX-resistant 
sodium channel $\mathrm{Na}_{\mathrm{v}} 1.8$ (Pinto et al., 2008), which secures spike generation during tonic depolarization and at cold temperatures (Zimmermann et al., 2007). This difference may well explain the lower-frequency heat response we observed in the axons compared with the endings. This argument, however, seems to contradict the axonal cold sensitivity we observed as TTX-sensitive sodium channels should be inactivated at cold temperatures, disabling action potential generation (Zimmermann et al., 2007). The cold-transducing mechanisms, perhaps involving TRP melastatin 8, TRPA1, or TREK-1/TRAAK (TWIK-related arachidonic acid-stimulated potassium channel) channels, may still be functional in the axonal membrane and enable cold-induced polarization. Because membrane ("input") resistance increases after cooling, the resulting generator current may be large enough to reach unblocked warmer parts of the axon (located beyond the sharp border of our metal ring), exceed the (low) threshold of $\mathrm{Na}_{\mathrm{V}} 1.7$, and trigger action potentials.

Our findings do not exclude the possibility that epineurial nerve endings (nervi nervorum) could mediate heat responsiveness of peripheral nerves (Sauer et al., 1999). To be compatible with our single-fiber recording results, this theory would require to assume an only heat-sensitive epineurial collateral of a fiber that proceeds through the nerve and terminates in a mechanoheat-sensitive RF of the skin. This assumption cannot currently be disproved, but appears feeble because heat-induced axonal CGRP release was previously discovered using desheathed peripheral nerves bare of epineurial nerve endings (Sauer et al., 2001).

The apparent rareness of heat-sensitizing BK effects on axonal responses (in contrast to Fischer and Reeh, 2007) is certainly attributable to the fact that we left the impermeable sheath of the nerve stem intact to prevent loss of fibers.

In conclusion, our findings establish axonal heat sensitivity and ectopic spike generation as a normal physiologic capacity in peripheral nerves. When exaggerated, this capacity may become a source of neuropathic pain if the heat threshold drops to body temperature, as may be the case during inflammation-like processes in the sick peripheral nerve. Cooling the nerve or pharmacological blocking of the heat transduction would then be a therapeutic option.

\section{References}

Alloui A, Zimmermann K, Mamet J, Duprat F, Noel J, Chemin J, Guy N, Blondeau N, Voilley N, Rubat-Coudert C, Borsotto M, Romey G, Heurteaux C, Reeh P, Eschalier A, Lazdunski M (2006) TREK-1, a K ${ }^{+}$channel involved in polymodal pain perception. EMBO J 25:2368-2376.

Baron R, Janig W, Kollmann W (1988) Sympathetic and afferent somata projecting in hindlimb nerves and the anatomical organization of the lumbar sympathetic nervous system of the rat. J Comp Neurol 275:460-468.

Bernardini N, Neuhuber W, Reeh PW, Sauer SK (2004) Morphological evidence for functional capsaicin receptor expression and calcitonin generelated peptide exocytosis in isolated peripheral nerve axons of the mouse. Neuroscience 126:585-590.

Blenk KH, Michaelis M, Vogel C, Janig W (1996) Thermosensitivity of acutely axotomized sensory nerve fibers. J Neurophysiol 76:743-752.
Devor M, Govrin-Lippmann R (1983) Axoplasmic transport block reduces ectopic impulse generation in injured peripheral nerves. Pain 16:73-85.

Dilley A, Bove GM (2008) Disruption of axoplasmic transport induces mechanical sensitivity in intact rat C-fiber nociceptor axons. J Physiol (Lond) 586:593-604.

Fischer MJ, Reeh PW (2007) Sensitization to heat through G-proteincoupled receptor pathways in the isolated sciatic mouse nerve. Eur J Neurosci 25:3570-3575.

Guharay F, Sachs F (1984) Stretch-activated single ion channel currents in tissue-cultured embryonic chick skeletal muscle. J Physiol (Lond) 352:685-701.

Koschorke GM, Meyer RA, Tillman DB, Campbell JN (1991) Ectopic excitability of injured nerves in monkey: entrained responses to vibratory stimuli. J Neurophysiol 65:693-701.

Koschorke GM, Meyer RA, Campbell JN (1994) Cellular components necessary for mechanoelectrical transduction are conveyed to primary afferent terminals by fast axonal transport. Brain Res 641:99-104.

Liang YF, Haake B, Reeh PW (2001) Sustained sensitization and recruitment of rat cutaneous nociceptors by bradykinin and a novel theory of its excitatory action. J Physiol (Lond) 532:229-239.

Michaelis M, Blenk KH, Janig W, Vogel C (1995) Development of spontaneous activity and mechanosensitivity in axotomized afferent nerve fibers during the first hours after nerve transection in rats. J Neurophysiol 74:1020-1027.

Michaelis M, Vogel C, Blenk KH, Janig W (1997) Algesics excite axotomised afferent nerve fibres within the first hours following nerve transection in rats. Pain 72:347-354.

Michaelis M, Blenk KH, Vogel C, Janig W (1999) Distribution of sensory properties among axotomized cutaneous C-fibres in adult rats. Neurosci Lett 94:7-10.

Moalem G, Grafe P, Tracey DJ (2005) Chemical mediators enhance the excitability of unmyelinated sensory axons in normal and injured peripheral nerve of the rat. Arthritis Rheum 134:1399-1411.

Mogil JS, Breese NM, Witty MF, Ritchie J, Rainville ML, Ase A, Abbadi N, Stucky CL, Seguela P (2005) Transgenic expression of a dominantnegative ASIC3 subunit leads to increased sensitivity to mechanical and inflammatory stimuli. J Neurosci 25:9893-9901.

Pinto V, Derkach VA, Safronov BV (2008) Role of TTX-sensitive and TTXresistant sodium channels in $\mathrm{A} \delta$ - and $\mathrm{C}$-fiber conduction and synaptic transmission. J Neurophysiol 99:617-628.

Reeh PW (1986) Sensory receptors in mammalian skin in an in vitro preparation. Neurosci Lett 66:141-147.

Sauer SK, Bove GM, Averbeck BA, Reeh PW (1999) Rat peripheral nerve components release calcitonin gene-related peptide and prostaglandin $\mathrm{E}_{2}$ in response to noxious stimuli: evidence that nervi nervorum are nociceptors. Neurosci 92:319-325.

Sauer SK, Reeh PW, Bove GM (2001) Noxious heat-induced CGRP release from rat sciatic nerve axons in vitro. Eur J Neurosci 14:1203-1208.

Schmelz M, Schmidt R, Ringkamp M, Handwerker HO, Torebjörg E (1995) Delayed responses to electrical stimuli reflect C-fiber responsiveness in human microneurography. Exp Brain Res 104:331-336.

Zimmermann K, Leffler A, Fischer MM, Messlinger K, Nau C, Reeh PW (2005) The TRPV1/2/3 activator 2-aminoethoxydiphenyl borate sensitizes native nociceptive neurons to heat in wildtype but not TRPV1 deficient mice. Neurosci 135:1277-1284.

Zimmermann K, Leffler A, Babes A, Cendan CM, Carr RW, Kobayashi J, Nau C, Wood JN, Reeh PW (2007) Sensory neuron sodium channel Nav1.8 is essential for pain at low temperatures. Nature 447:855-858.

Zimmermann M, Sanders KH (1982) Responses of nerve axons and receptor endings to heat, ischemia, and algesic substances. Abnormal excitability of regenerating nerve endings. In: Abnormal nerves and muscles as impulse generators (Culp WJ, Ochoa J, eds), pp 513-532. New York: Oxford UP. 